\title{
Evaluating and validating the predictive ability of preoperative systemic inflammatory/immune cells in gastric cancer following $\mathrm{RO}$ resection
}

\author{
WENBO XUE $^{1 *}$, XUEZHONG XU $^{1 *}$, YULIN TAN $^{1 *}$, YAN QIAN $^{2}, \mathrm{HAO} \mathrm{WANG}^{3}$, \\ YIBO WANG $^{1}$, YIXIN XU ${ }^{1}$, XIAOJUN ZHU ${ }^{1}$, PENG JIANG ${ }^{1}$ and WEI DING ${ }^{1}$ \\ ${ }^{1}$ Department of General Surgery, Wujin Hospital Affiliated to Jiangsu University, Changzhou, Jiangsu 213002; \\ ${ }^{2}$ Department of Respiration, Changzhou Second People's Hospital Affiliated to Nanjing Medical University, \\ Changzhou, Jiangsu 213164; ${ }^{3}$ Department of Medical Records, Wujin Hospital Affiliated to Jiangsu University, \\ Changzhou, Jiangsu 213002, P.R. China
}

Received February 11, 2019; Accepted August 29, 2019

DOI: $10.3892 / 01.2019 .10867$

\begin{abstract}
The present study aimed to compare the predictive abilities of preoperative systemic inflammatory/immune cell ratios in gastric cancer (GC) following curative R0 resection, and to screen the optimal parameter incorporated into nomograms to predict the postoperative overall survival (OS) and recurrence-free survival (RFS). A total of 679 patients with GC were included in the study, divided into a primary cohort (300 cases), an internal validation cohort (278 cases), and an external validation cohort (101 cases). In the primary cohort, the prognostic abilities of all systemic inflammatory/immune cell accounts or ratios were compared by receiver operating characteristic (ROC) curve analysis. The area under the ROC curve (AUC) of the neutrophil-monocyte-lymphocyte ratio (NMLR) was largest for the prediction of OS (AUC $=0.728)$ and RFS (AUC=0.695). The independent predictive factors for OS or RFS, including NMLR, degree of differentiation (DD),
\end{abstract}

Correspondence to: Mr. Wei Ding, Department of General Surgery, Wujin Hospital Affiliated to Jiangsu University, 2 Yong Ning Road North, Changzhou, Jiangsu 213002, P.R. China

E-mail: dwdoctor@163.com

*Contributed equally

Abbreviations: GC, gastric cancer; OS, overall survival; RFS, recurrence-free survival; TNM, tumor-nodes-metastasis; DD, degree of differentiation; NLR, neutrophil-to-lymphocyte ratio; MLR, monocyte-to-lymphocyte ratio; PLR, platelet-to-lymphocyte ratio; $\mathrm{CT}$, computed tomography; CEA, carcinoembryonic antigen; NMLR, neutrophil-monocyte-lymphocyte ratio; PNLR, plateletneutrophil-lymphocyte; PMLR, platelet-monocyte-lymphocyte; ROC, receiver operating characteristics; AUC, area under the ROC curve; HR, hazard ratio; CI, confidence interval

Key words: gastric cancer, prognostic, inflammatory cell, immune cell, gastrectomy
T-stage and $\mathrm{N}$-stage were used to establish the 2 nomograms. The comprehensive predictive power of nomograms was compared with that of the tumor-nodes-metastasis (TNM) staging system and validated by bootstrap resampling. The concordance indexes (C-indexes) of the nomograms for OS [C-index, 0.851; 95\% confidence interval (CI), 0.817-0.883] and RFS (C-index, 0.860; 95\% CI, 0.831-0.889), were increased compared with those for the DD, the NMLR and the TNM stage. The AUCs of the 2 nomograms ( 0.933 for OS and 0.944 for RFS) were largest among all predictive scoring systems. In the internal validation cohort, the $\mathrm{C}$-indexes of the nomograms for OS and RFS were 0.840 and 0.916 , respectively. In the external validation cohort, the C-indexes of the nomograms for OS and RFS nomograms were 0.827 and 0.891 , respectively. The present study demonstrated that the NMLR was an independent prognostic factor for patients with GC. The proposed nomograms were demonstrated to have a good predictive ability with improved sensitivity and accuracy in survival and recurrence in patients with GC undergoing $\mathrm{R} 0$ resection.

\section{Introduction}

Gastric cancer (GC) is one of the most common malignant tumor types according to previous statistics (1). The morbidity of GC ranks fourth and the associated mortality ranks second worldwide. Eastern Asia has the highest incidence of GC in the world. Despite comprehensive post-operative anti-tumor therapy resulting in prolonged survival following GC resection, long-term survival following surgery remains poor (2). Precise predictive tools are critical for individualizing treatment protocols. At present, the tumor-nodes-metastasis (TNM) stage is the most frequently used prognostic factor. However, clinical experience has indicated that even within the same TNM stage, the survival of patients may differ (3). Therefore, the development of novel evaluation systems that may include more prognostic factors is urgently required.

The predictive roles of the preoperative systemic inflammatory/immune cells in GC have been highlighted by 
previous studies, including the neutrophil-to-lymphocyte ratio (NLR), the monocyte-to-lymphocyte ratio (MLR) and the platelet-to-lymphocyte ratio (PLR) (4-6). Recently, the neutrophil-monocyte-lymphocyte ratio (NMLR) has been suggested to have an improved predictive ability compared with other inflammatory/immune cell counts or ratios in patients with hepatocellular carcinoma after curative hepatectomy (7). The most suitable parameter of the inflammatory/immune system for predicting the outcome for patients with $\mathrm{GC}$ remains to be determined.

To improve and refine the predictive models of traditional staging systems for GC, several novel nomograms have been reported $(8,9)$. These included prognostic nomograms for $\mathrm{GC}$ following gastrectomy incorporating systemic inflammatory/immune parameters. The present study performed a screening to identify the optimum systemic inflammatory/immune parameter and to develop reliable nomograms, in order to provide accurate estimations of the prognosis of patients with GC undergoing $\mathrm{R} 0$ resection.

\section{Patients and methods}

Ethics statement. The present retrospective cohort study was approved by the Ethics Review Committee of Wujin Hospital affiliated to Jiangsu University and was performed in accordance with the ethical guidelines of the Declaration of Helsinki from 1975. Due to the retrospective nature of this study, the need for written informed consent was waived.

Study population. Data were collected from Wujin Hospital and the Southern Branch of Wujin Hospital Affiliated to Jiangsu University. A total of 1,023 consecutive patients with GC confirmed by pathology undergoing radical gastrectomy were considered for the retrospective analysis The inclusion criteria were as follows: i) Detailed laboratory test data; ii) no pre-operative metastases confirmed by computed tomography (CT); iii) no pre-operative anti-tumor treatments; iv) complete lymph node dissection; v) complete records and follow-up data, and continuous regular follow-up. Finally, 679 patients were included into the present study and further divided into a primary cohort (January 2013 to December 2013; n=300), an internal validation cohort (January 2014 to October 2014; $\mathrm{n}=278$ ), and an external validation cohort (May 2012 to May 2015; $n=101$ ). The patients in the primary cohort and internal validation cohort were from Wujin Hospital and the patients in the external validation cohort were from the Southern Branch of Wujin Hospital. Wujin Hospital and the Southern Branch of Wujin Hospital are 2 different centers, independent of each other, serving different populations. Wujin Hospital serves the people $(\sim 1,300,000)$ from the Tianning, Zhonglou and Xinbei districts, and Changzhou city. The Southern Branch of Wujin Hospital serves the people $(\sim 1,400,000)$ from the Wujin district and Changzhou city. The grouping method was consistent with a previous study (7).

Data collection. Clinical characteristics, including the status of the patients, operative features, results of laboratory tests, histologic and pathologic features of tumors, and prognostic data were collected. The TNM staging system (American Joint Committee on Cancer, 8th ed., 2018) was used to stage the tumors (10). Laboratory examinations included neutrophil, lymphocyte, monocyte and platelet count, and D-dimer and carcinoembryonic antigen (CEA). The NLR was defined as the absolute neutrophil count divided by the absolute lymphocyte count. The MLR was defined as the absolute monocyte count divided by the absolute lymphocyte count. The PLR was defined as the absolute platelet count divided by the absolute lymphocyte count. The NMLR was defined as the product of the neutrophil count and monocyte count divided by the absolute lymphocyte count. The platelet-neutrophil-lymphocyte ratio (PNLR) was defined as the product of the platelet count and neutrophil count divided by the absolute lymphocyte count. The platelet-monocyte-lymphocyte ratio (PMLR) was defined as the product of platelet count and monocyte count divided by the absolute lymphocyte count.

Follow-up. During the first year following surgery, patients were examined once a month. During the second year, follow-up was performed every 3 months. For the third year, patients were followed up twice a year, and then once annually thereafter. The parameters determined at each visit included thoracic and abdominal CT scan, blood routine, hepatic and renal function, D-dimer and CEA.

Statistical analysis. The receiver operating characteristics (ROC) curve was used to calculate the optimal cutoff values (by Youden index) of systemic inflammatory/immune cell counts or ratios and the areas under the ROC curve (AUC). For continuous variables, differences between groups were analyzed using one-way analysis of variance with least significant difference test. Categorical variables were analyzed using the $\chi^{2}$ test. Survival curves were drawn using the Kaplan-Meier method and compared using log-rank tests. Parameters in nomograms were selected by univariate and multivariate analyses, using a Cox proportional-hazards model. Statistical analyses were performed with SPSS 20.0 for Windows (IBM Corp.).

Two nomograms were established using the rms package in R v.3.5.1 (http://www.r-project.org/). Differences between the predictive model and experimental data were quantified according to the concordance index (C-index). Bootstraps with 1,000 resamples were used to estimate bias and present calibration plots. For all statistical tests, a two-sided $\mathrm{P}<0.05$ was considered to indicate a statistically significant difference.

\section{Results}

Clinicopathological characteristics. The clinicopathological characteristics of the 679 cases in the primary and validation cohorts are summarized in Table I. The median follow-up time was 61, 51 and 49 months, the median age was 67, 66 and 67 years, and the median tumor size was $4.0,4.0$ and $3.5 \mathrm{~cm}$ in the primary, internal validation and external validation cohorts, respectively. Among all cases, the neutrophil, monocyte, lymphocyte and platelet counts ranged from $1.24-13.75 \times 10^{9} / 1$, $0.03-1.61 \times 10^{9} / 1,0.33-5.49 \times 10^{9} / 1$ and $68-768 \times 10^{9} / 1$, respectively. The laboratory test results were comparable among the three cohorts, with the exception of the monocyte $(\mathrm{P}=0.001)$, albumin $(\mathrm{P}<0.001)$ and globulin levels $(\mathrm{P}=0.001)$, as presented in Table I. 
Table I. Characteristics of patients in the primary and validation cohorts.

\begin{tabular}{|c|c|c|c|c|}
\hline Characteristics & $\begin{array}{l}\text { Primary cohort } \\
\qquad(n=300)\end{array}$ & $\begin{array}{l}\text { Internal validation } \\
\text { cohort }(n=278)\end{array}$ & $\begin{array}{l}\text { External validation } \\
\text { cohort }(n=101)\end{array}$ & P-value \\
\hline Age, year, median (range) & $67(39-91)$ & $66(38-85)$ & $67(29-92)$ & 0.363 \\
\hline Sex (male/female) & $214 / 86$ & $198 / 80$ & $78 / 23$ & 0.469 \\
\hline Neutrophil, 10x9/1, median (range) & $4.00(1.30-13.48)$ & $3.81(1.24-13.72)$ & $3.83(1.40-13.75)$ & 0.517 \\
\hline Monocyte, 10x9/1, median (range) & $0.35(0.03-1.23)$ & $0.39(0.11-1.27)$ & $0.41(0.07-1.61)$ & $<0.001$ \\
\hline Lymphocyte, 10x9/1, median (range) & $1.48(0.33-4.05)$ & $1.50(0.51-3.87)$ & $1.45(0.49-5.49)$ & 0.411 \\
\hline Platelet, 10x9/1, median (range) & $210(82-585)$ & $205(68-768)$ & $211(83-492)$ & 0.372 \\
\hline Albumin, median (range) & $40(22.3-50.3)$ & $39.7(24.39-59.3)$ & $42.8(26.6-55.6)$ & $<0.001$ \\
\hline Globulin, median (range) & $26.6(16.6-39.4)$ & $24.9(14.0-39.0)$ & $24.5(13.9-34.4)$ & $<0.001$ \\
\hline D-dimer, median (range) & $0.33(0.03-40.00)$ & $0.30(0.06-6.65)$ & $0.44(0.05-22.7)$ & 0.071 \\
\hline CEA, median (range) & $2.01(0.11-527.53)$ & $2.35(0.15-391.10)$ & $2.21(0.42-193.8)$ & 0.591 \\
\hline Tumor size, $\mathrm{cm}$ (range) & $4.0(0.3-14.0)$ & $4.0(0.3-14.0)$ & $3.5(0.6-11.0)$ & 0.172 \\
\hline Tumor differentiation (I-II/III-IV) & $52 / 248$ & $75 / 203$ & $15 / 86$ & 0.005 \\
\hline TNM stage (I-II/III) & $164 / 134$ & $153 / 125$ & $50 / 51$ & 0.589 \\
\hline
\end{tabular}

CEA, carcinoembryonic antigen.
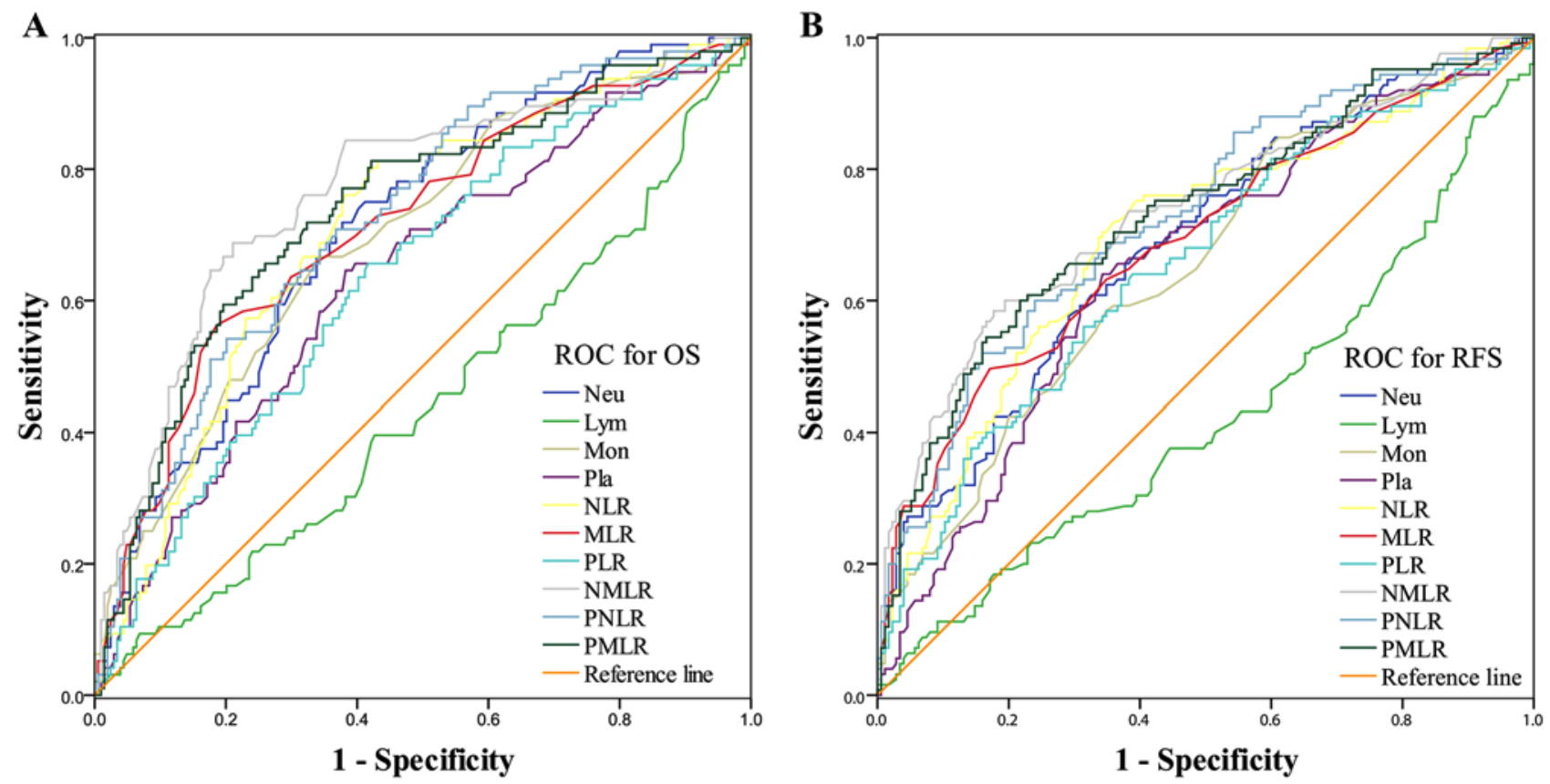

Figure 1. ROC curves of the prediction index values in predicting 3-year survival and 3-year overall recurrence-free survival of patients with gastric cancer. ROC curves were used to estimate the optimal cutoff values of systemic inflammatory/immune cell counts or ratios in (A) OS and (B) RFS. ROC, receiver operating characteristic; OS, overall survival; RFS, recurrence-free survival; Neu, neutrophils; Lym, lymphocytes; Mon, monocytes; Pla, platelets; NLR, neutrophil-to-lymphocyte ratio; MLR, monocyte-to-lymphocyte ratio; PLR, platelet-to-lymphocyte ratio; NMLR, neutrophil-monocyte-lymphocyte ratio; PNLR, platelet-neutrophil-lymphocyte ratio; PMLR, platelet-monocyte-lymphocyte ratio.

Overall survival (OS) and recurrence-free survival (RFS) in the three cohorts. For the primary cohort, the 1-, 3- and 5-year OS rates were 92.7, 68.0 and 57.7\%, and the 1-, 3- and 5-year RFS rates were 92.3, 58.3 and $39.7 \%$, respectively. For the internal validation cohort, the 1- and 3-year OS rates were 95.7 and $66.9 \%$; the 1- and 3-year RFS rates were 93.9 and $52.2 \%$, respectively. For the external validation cohort, the 1- and 3-year OS rates were 91.1 and $64.4 \%$, and the 1- and 3-year RFS rates were 90.1 and $48.5 \%$, respectively.
Comparison of predictive accuracy of the systemic inflammatory/immune parameters in the primary cohort. The optimal cutoff values of systemic inflammatory/immune cell counts or ratios were estimated from the ROC curves when the Youden index was maximal, as presented in Fig. 1. For OS, the optimal cutoff levels for the neutrophil, lymphocyte, monocyte and platelet counts, NLR, MLR, PLR, NMLR, PNLR and PMLR were 4.09, 1.10, 0.38, 216.00, 2.50, 0.29, 140.77, 1.15, 580.23 and 63.61, respectively. For RFS, the optimal cutoff 
A Inflammatory/immune cell counts or ratios

Overall survival

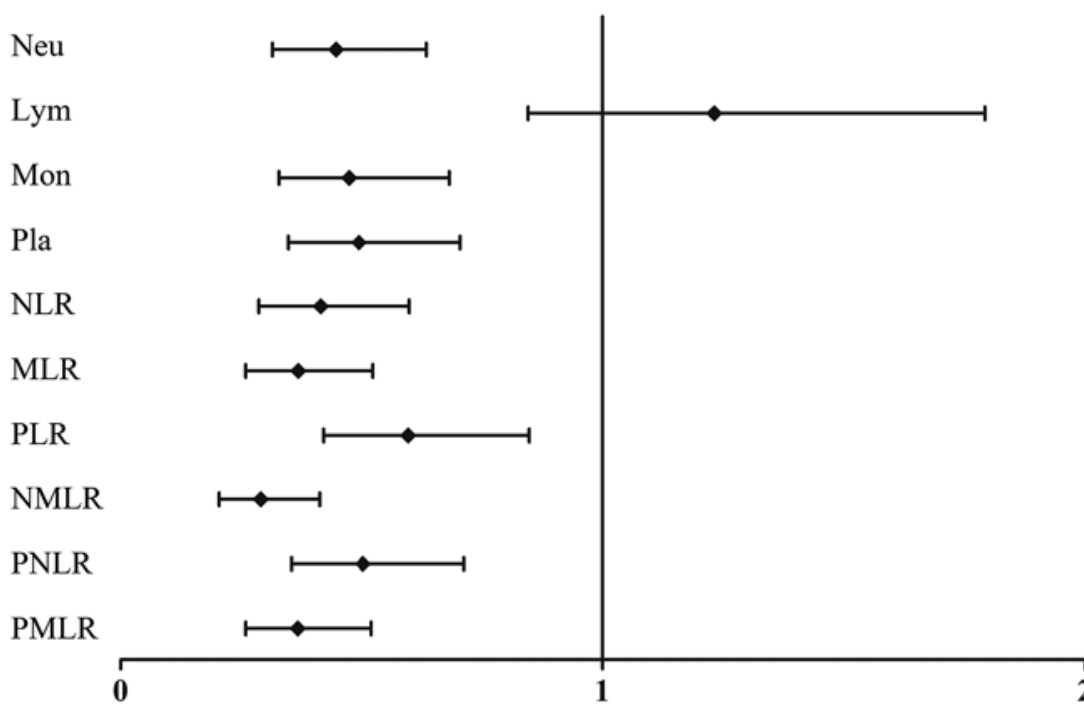

HR (95\% CI) P-value

0.447 (0.315-0.635) $\mathrm{P}<0.001$

$1.231(0.845-1.793) \mathrm{P}=0.280$

0.474 (0.329-0.682) $\mathrm{P}<0.001$

0.495 (0.348-0.704) $\mathrm{P}<0.001$

0.415 (0.287-0.599) $\mathrm{P}<0.001$

$0.369(0.260-0.524) \mathrm{P}<0.001$

$0.597(0.421-0.847) \mathrm{P}=0.004$

$0.291(0.205-0.413) \mathrm{P}<0.001$

0.503 (0.355-0.712) $\mathrm{P}<0.001$

$0.368(0.260-0.521) \mathrm{P}<0.001$

B

\section{Inflammatory/immune cell counts or ratios}

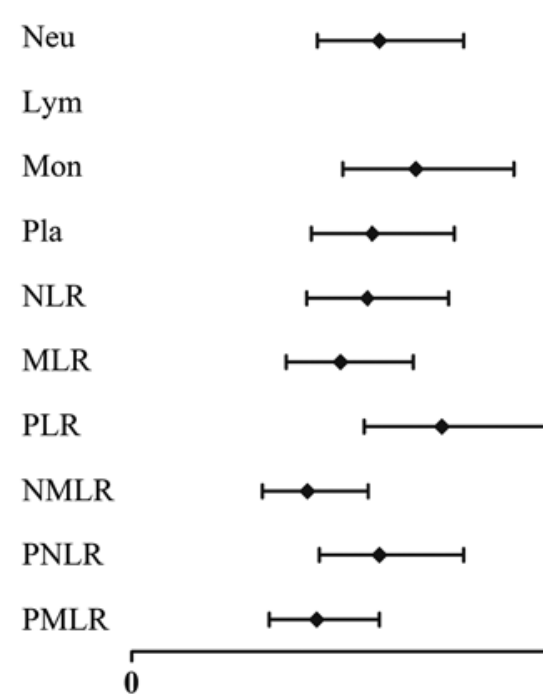

Recurrence-free survival

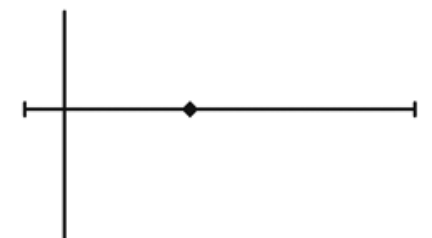

HR (95\% CI) P-value

0.515 (0.385-0.689) $\mathrm{P}<0.001$

$1.259(0.918-1.727) \mathrm{P}=0.153$

$0.590(0.438-0.793) \mathrm{P}<0.001$

$0.500(0.373-0.670) \mathrm{P}<0.001$

0.489 (0.363-0.658) $\mathrm{P}<0.001$

0.433 (0.321-0.585) $\mathrm{P}<0.001$

$0.644(0.482-0.861) \mathrm{P}=0.003$

$0.365(0.272-0.490) \mathrm{P}<0.001$

0.515 (0.389-0.689) $\mathrm{P}<0.001$

$0.384(0.286-0.515) \mathrm{P}<0.001$

Figure 2. Association between the systemic inflammatory/immune parameters and prognosis. HR and CI of (A) overall survival and (B) recurrence-free survival rates were analyzed using the log-rank method for the systemic inflammatory/immune cells counts and ratios. HR, hazard ratio; CI, confidence interval; Neu, neutrophils; Lym, lymphocytes; Mon, monocytes; Pla, platelets; NLR, neutrophil-to-lymphocyte ratio; MLR, monocyte-to-lymphocyte ratio; PLR, platelet-to-lymphocyte ratio; NMLR, neutrophil-monocyte-lymphocyte ratio; PNLR, platelet-neutrophil-lymphocyte ratio; PMLR, platelet-monocytelymphocyte ratio.

levels for the neutrophil, lymphocyte, monocyte and platelet counts, NLR, MLR, PLR, NMLR, PNLR and PMLR were $4.38,1.28,0.29,216.00,2.65,0.29,144.29,1.17,668.00$ and 59.22 , respectively. The final cut-off levels for the neutrophil, lymphocyte, monocyte and platelet counts, NLR, MLR, PLR, NMLR, PNLR and PMLR were calculated as the average of the OS and RFS values, and were set as 4.23, 1.19, 0.33, 216.00, $2.57,0.29,143.00,1.16,624.00$ and 61.40 , respectively.

The quality of the association between each systemic inflammatory/immune parameter and the OS or RFS rates were compared using log-rank tests. As presented in Fig. 2, the neutrophil count (both $\mathrm{P}<0.001$ ), monocyte count (both $\mathrm{P}<0.001$ ), platelet count (both $\mathrm{P}<0.001$ ), NLR (both $\mathrm{P}<0.001$ ), MLR (both $\mathrm{P}<0.001)$, PLR $(\mathrm{P}=0.004$ and $\mathrm{P}=0.003)$, NMLR (both $\mathrm{P}<0.001$ ), PNLR (both $\mathrm{P}<0.001$ ) and PMLR (both $\mathrm{P}<0.001)$ were associated with OS and RFS. The sensitivities and specificities of each parameter was compared using ROC curves, as presented in Fig. 3. Among all inflammatory/immune parameters, the NMLR consistently exhibited the highest AUC value for OS (AUC=0.728) and RFS (AUC=0.695). Patients with a low NMLR demonstrated significantly improved OS and RFS compared with those with a high NMLR, as presented in Fig. 4. 
A

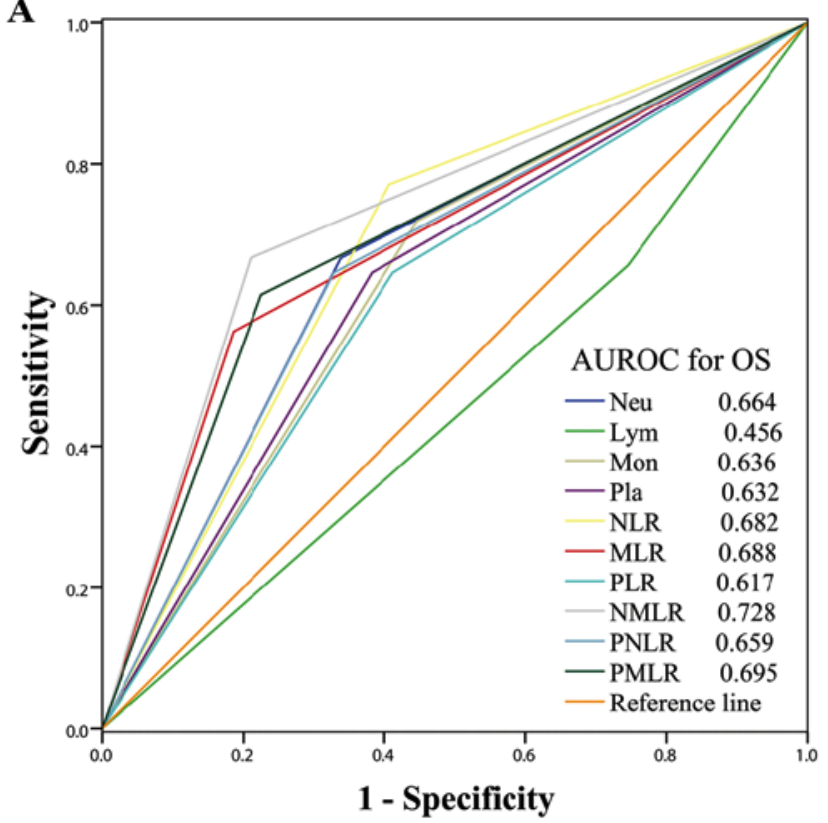

B

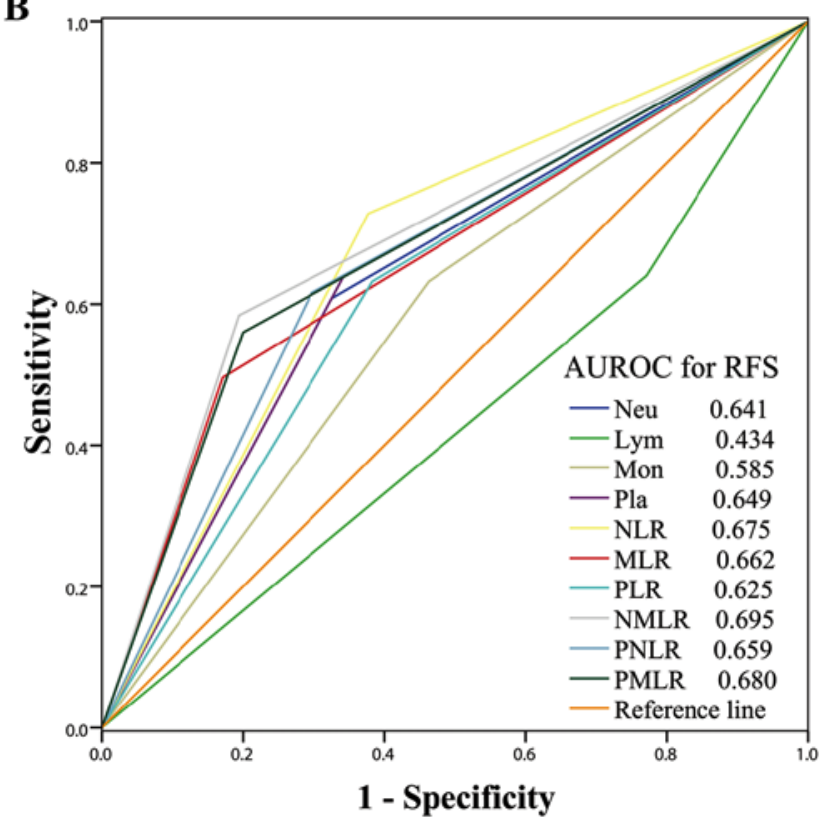

Figure 3. Predictive accuracy comparison of the systemic inflammatory/immune parameters for prognosis. AUROC was used to compare the sensitivities and specificities of the systemic inflammatory/immune cells counts and ratios to (A) OS or (B) RFS. AUROC, area under the receiver operating characteristic curve; OS, overall survival; RFS, recurrence-free survival; Neu, neutrophils; Lym, lymphocytes; Mon, monocytes; Pla, platelets; NLR, neutrophil-to-lymphocyte ratio; MLR, monocyte-to-lymphocyte ratio; PLR, platelet-to-lymphocyte ratio; NMLR, neutrophil-monocyte-lymphocyte ratio; PNLR, platelet-neutrophil-lymphocyte ratio; PMLR, platelet-monocyte-lymphocyte ratio.

A

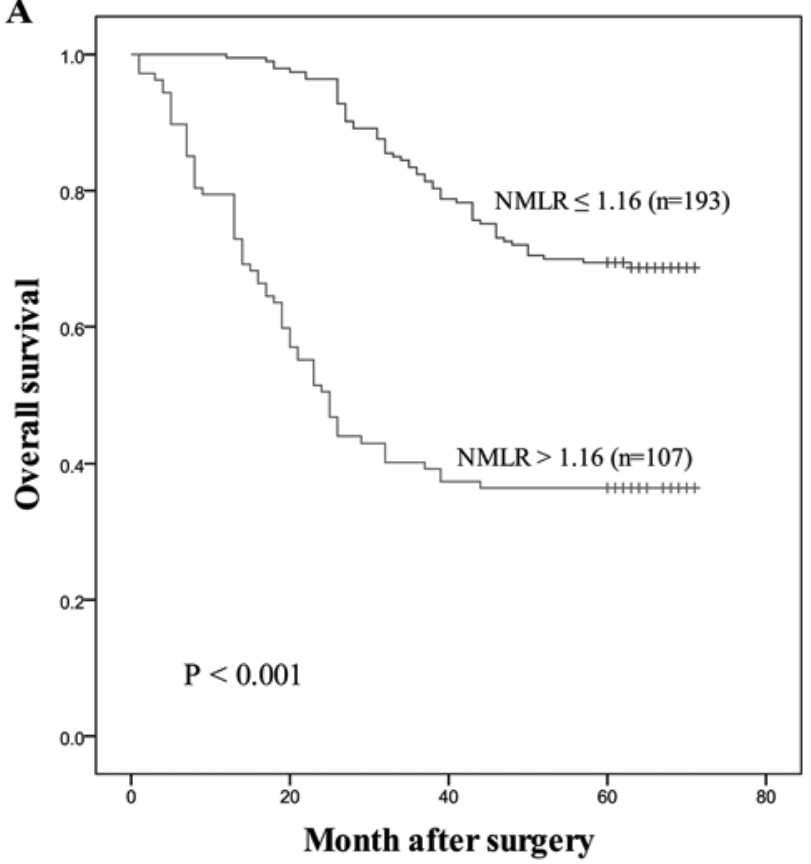

B

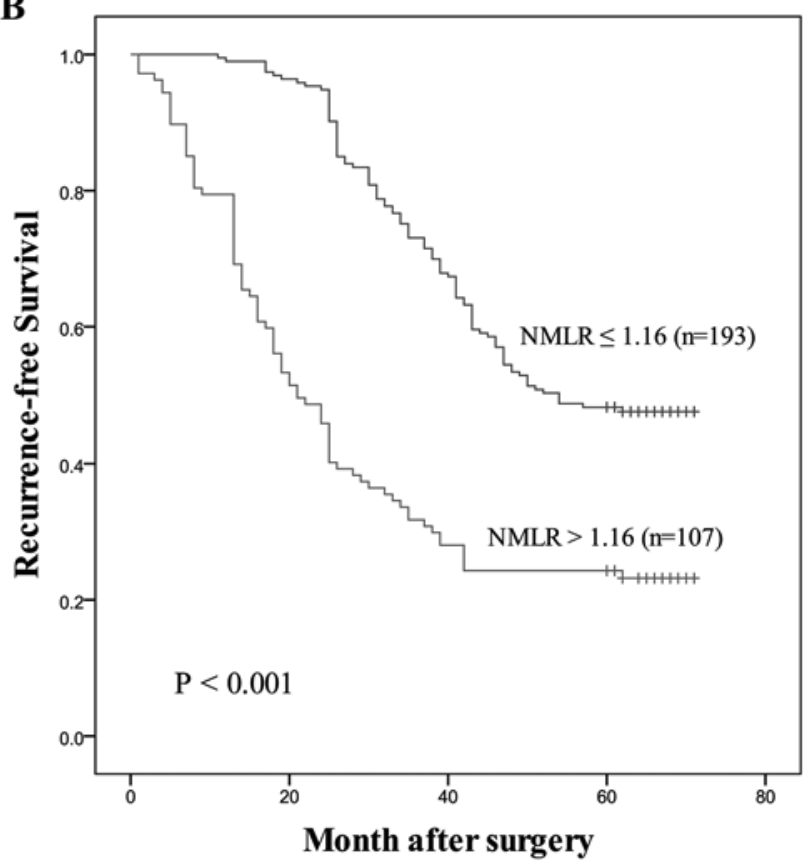

Figure 4. Survival curve for patients with low NMLR and high NMLR in the primary cohort. Associations between the NMLR and the (A) overall survival or (B) recurrence-free survival rates were compared using the Kaplan-Meier method. NMLR, neutrophil-monocyte-lymphocyte ratio.

Prognostic factors according to univariate and multivariate analyses in the primary cohort. In the primary cohort, univariate analyses were used to identify the potential predictive factors. Subsequently, the significant predictive factors were subjected to multivariate analyses. It was demonstrated that the degree of differentiation (DD; $\mathrm{P}=0.003$ and $\mathrm{P}=0.010$ ), $\mathrm{T}$ stage (both $\mathrm{P}<0.001$ ), $\mathrm{N}$ stage (both $\mathrm{P}<0.001$ ), and NMLR (both $\mathrm{P}<0.001$ ) were independent prognostic factors for OS and RFS, respectively (Table II).

Establishment and evaluation of nomograms for OS and RFS. The nomograms for OS (Fig. 5A) and RFS (Fig. 5B) were established using independent prognostic factors identified in the multivariate analysis conducted in the primary cohort. The 
Table II. Univariate and multivariate analyses, using a Cox proportional-hazards model of OS and recurrence-free survival of gastric cancer in primary cohort.

\begin{tabular}{|c|c|c|c|c|c|c|c|c|}
\hline \multirow{3}{*}{$\begin{array}{l}\text { Prognostic } \\
\text { variables }\end{array}$} & \multicolumn{4}{|c|}{ OS } & \multicolumn{4}{|c|}{ RFS } \\
\hline & \multicolumn{2}{|c|}{ Univariate analysis } & \multicolumn{2}{|c|}{ Multivariate analysis } & \multicolumn{2}{|c|}{ Univariate analysis } & \multicolumn{2}{|c|}{ Multivariate analysis } \\
\hline & $\mathrm{HR}(95 \% \mathrm{CI})$ & P-value & HR (95\% CI) & P-value & $\mathrm{HR}(95 \% \mathrm{CI})$ & $\mathrm{P}$-value & HR $(95 \%$ CI) & P-value \\
\hline Age & $\begin{array}{c}0.998 \\
(0.979-1.017)\end{array}$ & 0.833 & - & - & $\begin{array}{c}1.004 \\
(0.988-1.020)\end{array}$ & 0.620 & - & - \\
\hline Sex & $\begin{array}{c}1.532 \\
(1.007-2.330)\end{array}$ & 0.046 & - & - & $\begin{array}{c}1.082 \\
(0.919-1.274)\end{array}$ & 0.345 & - & - \\
\hline Tumor size & $\begin{array}{c}1.161 \\
(1.089-1.239)\end{array}$ & $<0.001$ & - & - & $\begin{array}{c}1.181 \\
(1.120-1.245)\end{array}$ & $<0.001$ & - & - \\
\hline DD & - & $<0.001$ & - & 0.003 & - & $<0.001$ & - & 0.010 \\
\hline T-stage & - & $<0.001$ & - & $<0.001$ & - & $<0.001$ & - & $<0.001$ \\
\hline $\mathrm{N}$-stage & - & $<0.001$ & - & $<0.001$ & - & $<0.001$ & - & $<0.001$ \\
\hline NMLR & $\begin{array}{c}0.291 \\
(0.205-0.413)\end{array}$ & $<0.001$ & $\begin{array}{c}0.162 \\
(0.107-0.244)\end{array}$ & $<0.001$ & $\begin{array}{c}0.365 \\
(0.272-0.490)\end{array}$ & $<0.001$ & $\begin{array}{c}0.229 \\
(0.165-0.318)\end{array}$ & $<0.001$ \\
\hline D-dimer & $\begin{array}{c}0.984 \\
(0.918-1.054)\end{array}$ & 0.639 & - & - & $\begin{array}{c}1.021 \\
(0.977-1.066)\end{array}$ & 0.356 & - & - \\
\hline CEA & $\begin{array}{c}1.003 \\
(1.000-1.005)\end{array}$ & 0.025 & - & - & $\begin{array}{c}1.003 \\
(1.000-1.005)\end{array}$ & 0.033 & - & - \\
\hline
\end{tabular}

OS, overall survival; RFS, recurrence-free survival; HR, hazard ratio; CI, confidence interval; DD, degree of differentiation; NLR, neutrophil-to-lymphocyte ratio; MLR, monocyte-to-lymphocyte ratio; PLR, platelet-to-lymphocyte ratio; NMLR, neutrophil-monocyte-lymphocyte ratio; PNLR, platelet-neutrophil-lymphocyte ratio; PMLR, platelet-monocyte-lymphocyte ratio; CEA, carcinoembryonic antigen.

C-index of the nomograms for OS was 0.851 [95\% confidence interval (CI), 0.817-0.883], which was increased compared with that of DD $(0.636 ; 95 \% \mathrm{CI}, 0.593-0.679)$, the NMLR $(0.667 ; 95 \% \mathrm{CI}, 0.628-0.706)$ and the TNM stage $(0.731 ; 95 \%$ CI, 0.698-0.764). The C-index of the nomograms for RFS was 0.860 (95\% CI, 0.831-0.889), which was increased compared with that of DD $(0.629 ; 95 \% \mathrm{CI}, 0.591-0.667)$, the NMLR $(0.645 ; 95 \%$ CI, $0.612-0.678)$ and the TNM stage $(0.740 ; 95 \%$ CI, 0.712-0.768). Concomitantly, the nomograms for OS and RFS exhibited the largest AUC value ( 0.933 for OS and 0.944 for RFS) compared with DD ( 0.674 for OS and 0.679 for RFS), NMLR (0.728 for OS and 0.695 for RFS) and TNM stage (0.817 for OS and 0.855 for RFS), as presented in Fig. 6. In the internal validation cohort, the C-indexes of the nomogram for OS and RFS were 0.840 (95\% CI, 0.803-0.877) and 0.916 (95\% CI, 0.895-0.937), respectively. In the external validation cohort, the C-indexes of the nomogram for OS and RFS were 0.827 (95\% CI, 0.763-0.891) and 0.891 (95\% CI, 0.852-0.930), respectively. The calibration plots generated in the present study exhibited a good coherence between the predictions and observations regarding 3-year survival and recurrence, as presented in Fig. 7.

\section{Discussion}

In the development of GC, sustained inflammatory and immune responses are hypothesized to serve a role, and they are considered to be the most important risk factors for prognosis $(11,12)$. GC is associated with Helicobacter pylori infection, which stimulates Toll-like receptors, induces infection-associated inflammation and generates an inflammatory microenvironment by activating innate immunity. Immune cells, particularly regulatory $\mathrm{T}$ cells, have been considered to be involved in inflammatory and immune response during the development of GC (13). These responses result in neutrophilia, lymphopenia and thrombocytosis. A high absolute neutrophil, monocyte and platelet count, and a low absolute count of lymphocytes have been demonstrated to be associated with poor prognosis of patients with GC $(14,15)$. The tumor immune microenvironment of GC is complex and changeable, and it involves various inflammatory cells, immune cells and tumor cells. The majority of single inflammatory or immune cell type counts are not sufficient to predict the prognosis of patients with GC after R0 resection. Numerous studies have revealed that systemic inflammation/immune cell ratios may be recognized as significant independent risk factors for the prognosis of GC (5,16-18). However, to date, only a few studies have compared the predictive value of all inflammatory/immune parameters $(7,19)$. The present study demonstrated that the NMLR exhibited the highest accuracy and predictive power; among all inflammatory/immune parameters assessed, it was the only parameter that was independently associated with OS and RFS.

The roles of inflammatory and immune cells in tumorigenesis may explain their predictive capacities regarding prognosis. Increasing evidence suggests that inflammatory environments accelerate the progression of metastasis by neutrophil-mediated mechanisms (20). For example, neutrophils contribute to the 
A

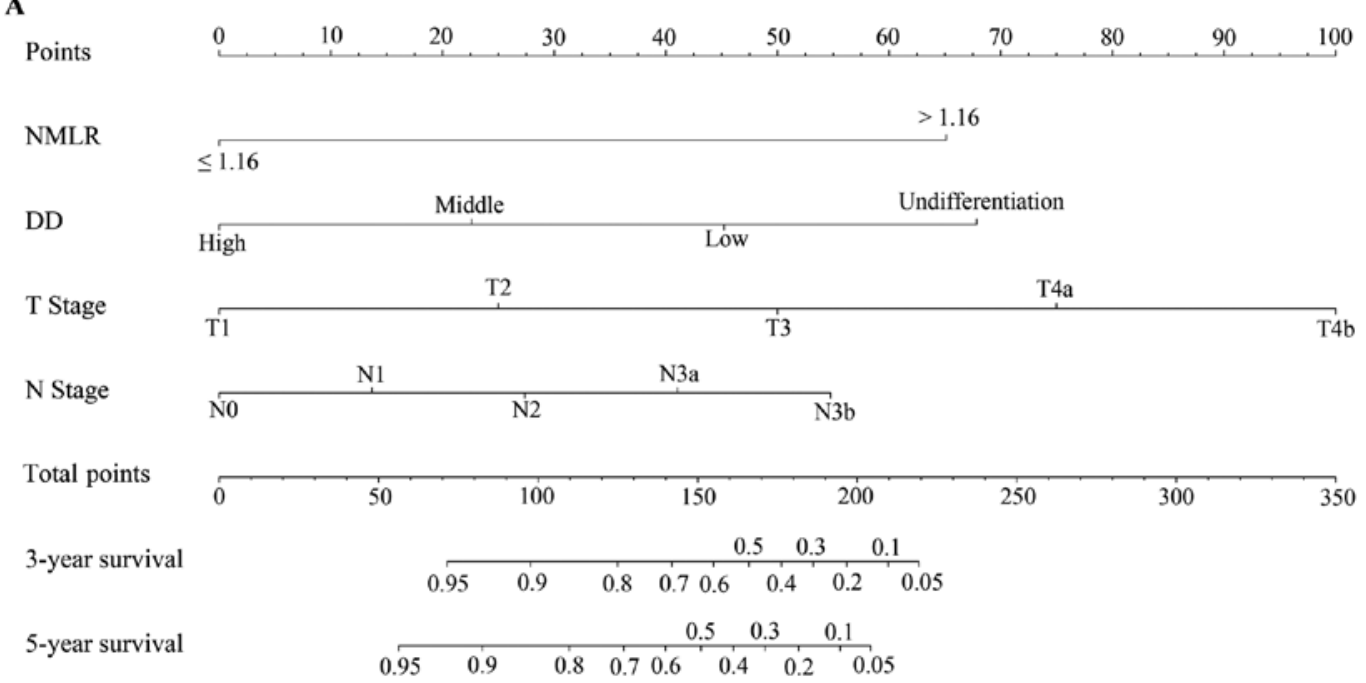

B

Points

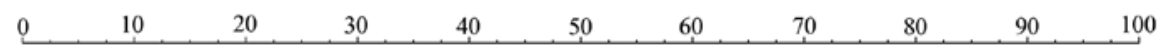

NMLR

$>1.16$

DD

$\begin{array}{llll}\leq 1.16 & & \\ & & & \\ \text { High } & \text { Middle } & \text { Undifferentiation } & \\ & & \end{array}$

T Stage

$\mathrm{T} 2$

T1

T4a

N Stage

N1

N3a

$\sqrt{\mathrm{N}}+\mathrm{N} 2 \quad \mathrm{~N} 3 \mathrm{~b}$

Total points

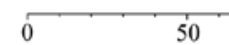

$100 \cdots$

$200-300-350$

3-year RFS

5-year RFS

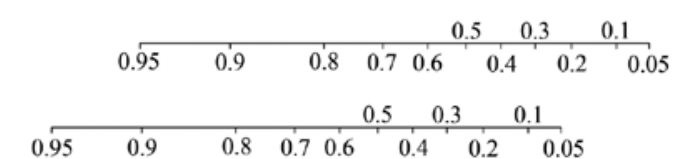

Figure 5. Development of novel nomograms for gastric cancer prognosis incorporating the systemic inflammatory/immune parameters. Nomograms for the prediction of the 3- and 5-year (A) overall survival and (B) recurrence-free survival in the primary cohort. NMLR, neutrophil-monocyte-lymphocyte ratio; $\mathrm{DD}$, degree of differentiation.
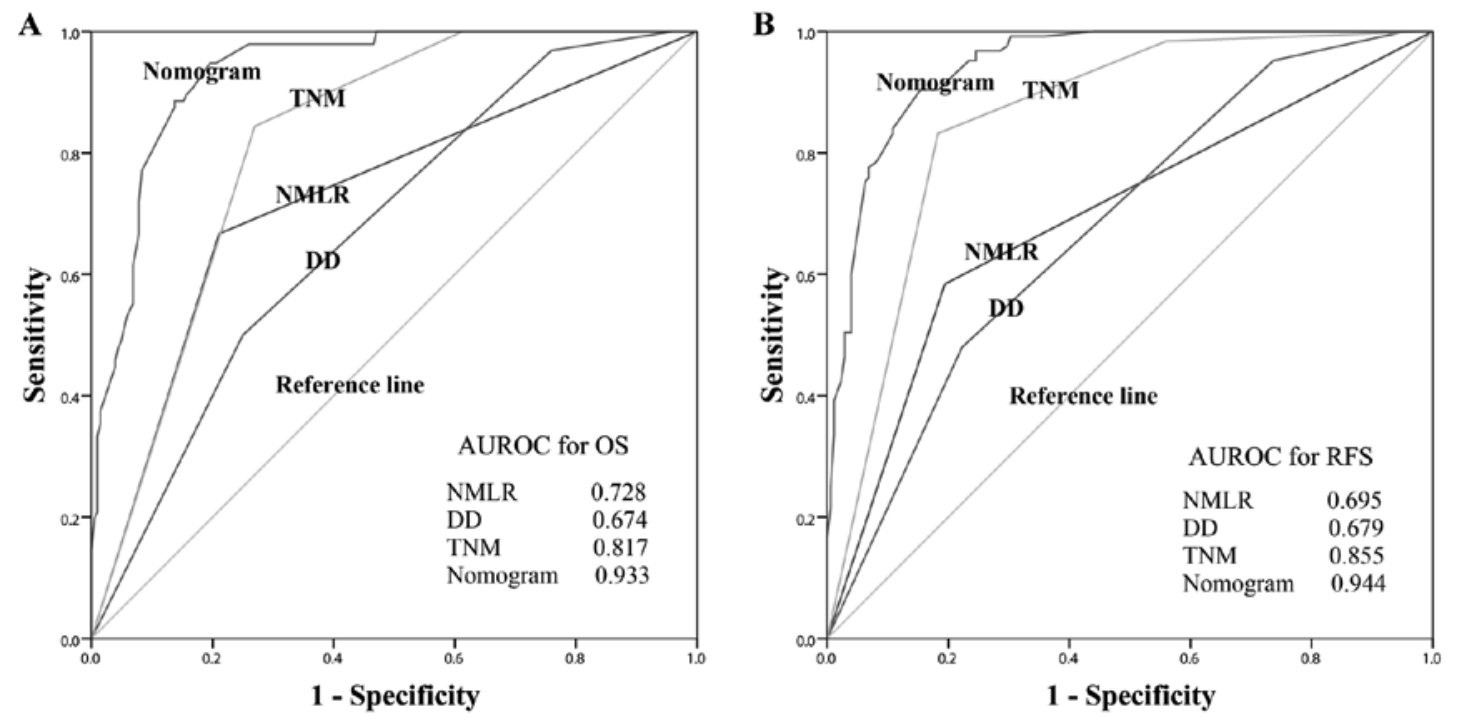

Figure 6. Predictive accuracy comparison of the each prediction systems for prognosis. Comparison of the predictive accuracy of each variable included in the (A) OS and (B) RFS nomograms by ROC curve analyses. TNM, tumor node metastasis; NMLR, neutrophil-monocyte-lymphocyte ratio; DD, degree of differentiation; AUROC, area under the receiver operating characteristic curve; OS, overall survival; RFS, recurrence-free survival. 
Nomogram-predicted probability of 3-year OS
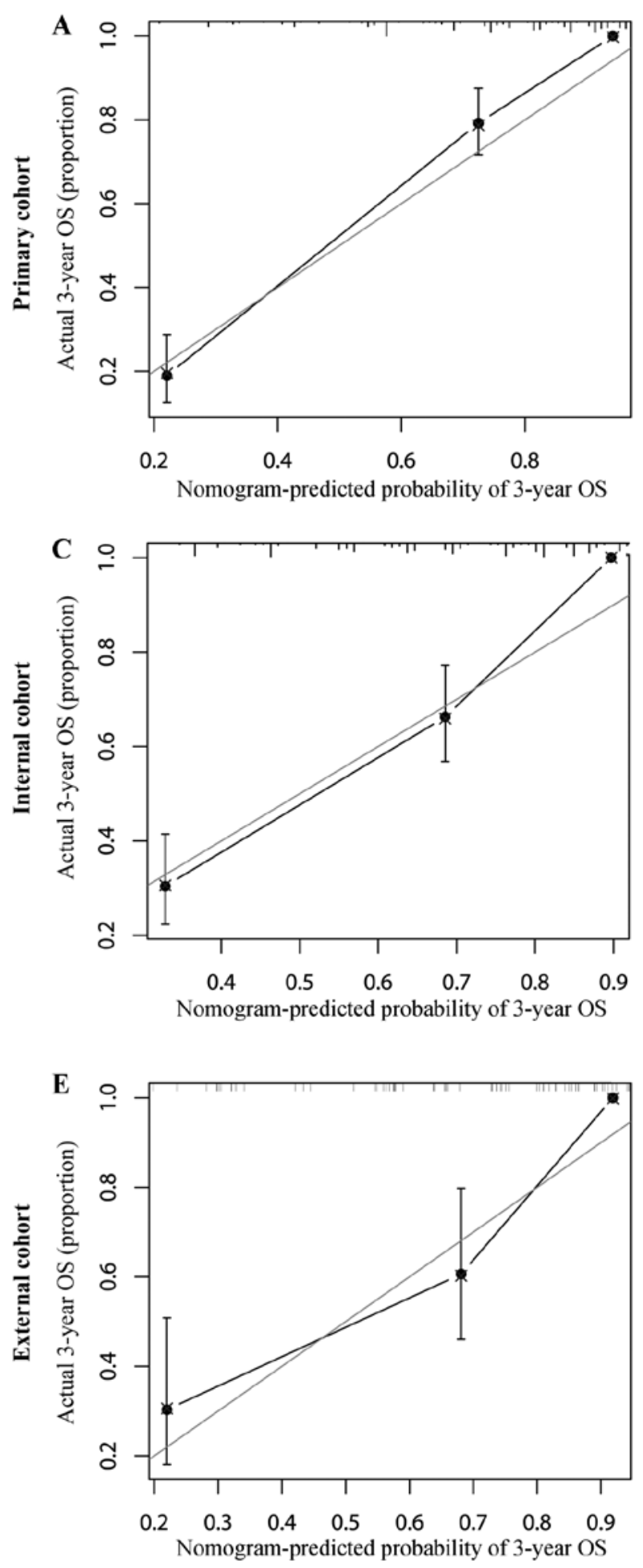

Nomogram-predicted probability of 3-year RFS
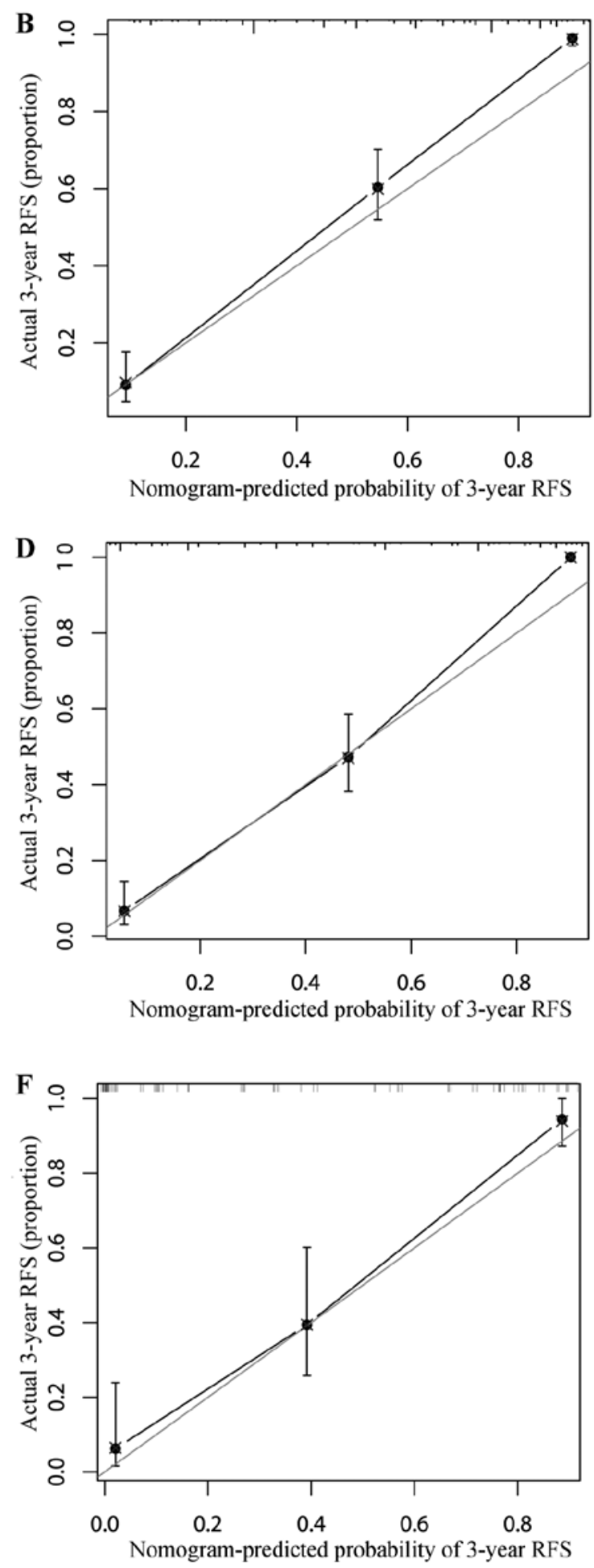

Figure 7. Calibration curves for predicting the 3-year OS and RFS rates by nomogram prediction and actual observation in patients with gastric cancer in the primary, internal validation and external validation cohorts. (A) 3-year OS prediction in the primary cohort. (B) 3-year RFS prediction in the primary cohort. (C) 3-year OS prediction in the internal validation cohort. (D) 3-year RFS prediction in the internal validation cohort. (E) 3-year OS prediction in the external validation cohort. (F) 3-year RFS prediction in the external validation cohort. OS, overall survival; RFS, recurrence-free survival.

initiation of natural killer cell and monocyte recruitment by various mechanisms (21). Neutrophils and monocytes may contribute to tumor progression by releasing prostaglandin E2 to amplify inflammation and create the tumor microenvironment (22). Conversely, lymphocytes may kill tumor cells through cytotoxic effects from the release of chemokines and cytokines, including interleukin-16, C-C motif chemokine ligand 21 and vascular endothelial growth factor $\mathrm{A}$, which attract monocytes, dendritic cells and endothelial cells to the tumor core and invasive margin $(23,24)$. Therefore, the NMLR may reflect the complex interaction between neutrophils, monocytes and lymphocytes in the tumor microenvironment. 
The present study analyzed the predictive ability of NMLR. To the best of our knowledge, the present study was the first to demonstrate that the NMLR, which reflects the homeostasis between host inflammatory and immune status, exhibited a greater prognostic value in GC compared with any other inflammatory/immune parameters; it was also the first to demonstrate that 2 specific OS and RFS nomograms, which included NMLR as one of their factors, exhibited high predictive values compared with measuring NMLR and the TNM stage separately. Among all factors involved in the 2 nomograms, the $\mathrm{T}$ stage, $\mathrm{N}$ stage and DD have been previously suggested to be associated with the prognosis of GC after gastrectomy $(25,26)$. Although certain risk factors, including CEA, sex, age and D-dimer, are associated with the prognosis of patients with GC (27-30), these factors were not applicable in the present study.

The nomogram described in the present study has several specific characteristics that distinguish it from previous nomograms. Firstly, the clinical and pathological factors included in the nomograms of the present study are much simpler to determine by routine clinical analysis. Furthermore, the nomogram did not just include the severity of GC, but the immune status of the patient was also considered. Finally, internal and external validation confirmed this accuracy.

There are several limitations which should be taken into consideration when interpreting the conclusions of the present study. Firstly, the present study was limited by its retrospective nature. Furthermore, as certain cases were followed up for $<5$ years, the 5-year survival rate and 5-year recurrence-free survival rate were not sufficiently accurate. In addition, the effects of adjuvant treatment, including chemotherapy or radiation treatment, were not evaluated. As an additional limitation, nutritive indexes were not considered in the present study. Previous studies demonstrated that certain nutritive indexes, including Controlling Nutritional Status, prognostic nutritional index and pre-operative body weight, were closely associated with the prognosis of GC (31-33). Finally, comorbidities, including hypertension and diabetes, were not reflected in the nomograms. It may be assumed that comorbidities may affect the prognosis to a certain extent.

In conclusion, 2 nomograms were described in the present study, which demonstrated predictive value for survival and recurrence in patients with GC after R0 resection with improved sensitivity and accuracy. This evaluation system may provide valuable insight into identifying patients with a high risk of poor prognosis following surgery. Close follow-up and comprehensive anti-tumor therapy are more suitable for these people. However, a large-sample prospective study is required to determine whether these nomograms are sufficiently accurate, and whether any further risk factors should be considered for inclusion in the assessment.

\section{Acknowledgments}

Not applicable.

\section{Funding}

The present study received a grant (grant no. WS201515) from the Science and Technology Bureau of Changzhou Municipal Wujin District.

\section{Availability of data and materials}

The datasets used and/or analyzed during the current study are available from the corresponding author on reasonable request.

\section{Authors' contributions}

YT, HW, YW and PJ were responsible for data curation and YQ performed the statistical analysis. XX was responsible for acquiring the funding. WD, $\mathrm{XX}$ and $\mathrm{XZ}$ developed the methodology of the present study and WD, WX and YX performed the software analysis. WD and XX supervised the study. WD and WX wrote the original draft of the manuscript, and WD performed the review and editing of the manuscript.

\section{Ethics approval and consent to participate}

The present study was approved by the Ethics Committee of Wujin Hospital Affiliated to Jiangsu University. Due to the retrospective nature of this study, the need for written informed consent was waived.

\section{Patient consent for publication}

Due to the retrospective nature of this study, informed consent was waived.

\section{Competing interests}

The authors declare that they have no competing interests.

\section{References}

1. Torre LA, Bray F, Siegel RL, Ferlay J, Lortet-Tieulent J and Jemal A: Global cancer statistics, 2012. CA Cancer J Clin 65: 87-108, 2015.

2. Fornaro L, Vasile E, Aprile G, Goetze TO, Vivaldi C, Falcone A and Al-Batran SE: Locally advanced gastro-oesophageal cancer: Recent therapeutic advances and research directions. Cancer Treat Rev 69: 90-100, 2018.

3. Shah MA and Ajani JA: Gastric cancer-an enigmatic and heterogeneous disease. JAMA 303: 1753-1754, 2010.

4. Arigami T, Uenosono Y, Matsushita D, Yanagita S, Uchikado Y, Kita Y, Mori S, Kijima Y, Okumura H, Maemura K, et al: Combined fibrinogen concentration and neutrophil-lymphocyte ratio as a prognostic marker of gastric cancer. Oncol Lett 11: 1537-1544, 2016.

5. Chen L, Hao Y, Zhu L, Li S, Zuo Y, Zhang Y, Song H and Xue Y: Monocyte to lymphocyte ratio predicts survival in patients with advanced gastric cancer undergoing neoadjuvant chemotherapy. Onco Targets 10: 4007-4016, 2017

6. Zhang Y, Lu JJ, Du YP, Feng CX, Wang LQ and Chen MB: Prognostic value of neutrophil-to-lymphocyte ratio and platelet-to-lymphocyte ratio in gastric cancer. Medicine (Baltimore) 97: e0144, 2018.

7. Liao R, Peng C, Li M, Li DW, Jiang N, Li PZ, Ding X, Wu Q, Du CY and Gong JP: Comparison and validation of the prognostic value of preoperative systemic immune cells in hepatocellular carcinoma after curative hepatectomy. Cancer Med 7: 1170-1182, 2018.

8. Zheng ZF, Lu J and Huang CM: ASO author reflections: Simplified nomogram predictive of survival after R0 resection for gastric cancer. Ann Surg Oncol 25 (Suppl 3): S733-S734, 2018.

9. Liu X, Wu Z, Lin E, Li W, Chen Y, Sun X and Zhou Z: Systemic prognostic score and nomogram based on inflammatory, nutritional and tumor markers predict cancer-specific survival in stage II-III gastric cancer patients with adjuvant chemotherapy. Clin Nutr 38: 1853-1860, 2019. 
10. Ajani JA, In H, Sano T, et al: Stomach. In: Amin MB (ed). AJCC cancer staging manual, 8th edition. New York, Springer-Verlag, 2016.

11. Matsueda S and Graham DY: Immunotherapy in gastric cancer. World J Gastroenterol 20: 1657-1666, 2014

12. Echizen K, Hirose O, Maeda Y and Oshima M: Inflammation in gastric cancer: Interplay of the COX-2/prostaglandin E2 and Toll-like receptor/MyD88 pathways. Cancer Sci 107: 391-397, 2016.

13. Kandulski A, Malfertheiner $\mathrm{P}$ and Wex T: Role of regulatory T-cells in $\mathrm{H}$. pylori-induced gastritis and gastric cancer. Anticancer Res 30: 1093-1103, 2010.

14. Shen Q, Liu W, Quan H, Pan S, Li S, Zhou T, Ouyang Y and Xiao H: Prealbumin and lymphocyte-based prognostic score, a new tool for predicting long-term survival after curative resection of stage II/III gastric cancer. Br J Nutr 120: 1359-1369, 2018.

15. Feng F, Zheng G and Wang Q: Low lymphocyte count and high monocyte count predicts poor prognosis of gastric cancer. BMC Gastroenterol 18: 148, 2018.

16. Kim H, Ro SM, Yang JH, Jeong JW, Lee JE, Roh SY and Kim IH: The neutrophil-to-lymphocyte ratio prechemotherapy and postchemotherapy as a prognostic marker in metastatic gastric cancer. Korean J Intern Med 33: 990-999, 2018.

17. Ma M, Wang J, Hu Y, Weng M, Liu X and Wang Y: Prognostic value of inflammatory biomarkers in gastric cancer patients and the construction of a predictive model. Dig Surg 36: 433-442, 2019.

18. Wen J, Bedford M, Begum R, Mitchell H, Hodson J, Whiting J and Griffiths $E$ : The value of inflammation based prognostic scores in patients undergoing surgical resection for oesophageal and gastric carcinoma. J Surg Oncol 117: 1697-1707, 2018.

19. Zhou ZQ, Pang S, Yu XC, Xue Q, Jiang HY, Liang XJ and Liu L: Predictive values of postoperative and dynamic changes of inflammation indexes in survival of patients with resected colorectal cancer. Curr Med Sci 38: 798-808, 2018.

20. McDonald B, Spicer J, Giannais B, Fallavollita L, Brodt P and Ferri LE: Systemic inflammation increases cancer cell adhesion to hepatic sinusoids by neutrophil mediated mechanisms. Int J Cancer 125: 1298-1305, 2009.

21. Nowarski R, Gagliani N, Huber S and Flavell RA: Innate immune cells in inflammation and cancer. Cancer Immunol Res 1: 77-84, 2013.

22. Spiegel A, Brooks MW, Houshyar S, Reinhardt F, Ardolino M, Fessler E, Chen MB, Krall JA, DeCock J, Zervantonakis IK, et al: Neutrophils suppress intraluminal NK cell-mediated tumor cell clearance and enhance extravasation of disseminated carcinoma cells. Cancer Discov 6: 630-649, 2016.

23. He W, Zhang H, Han F, Chen X, Lin R, Wang W, Qiu H, Zhuang Z, Liao Q, Zhang W, et al: CD155T/TIGIT signaling regulates CD ${ }^{+}$ T-cell metabolism and promotes tumor progression in human gastric cancer. Cancer Res 77: 6375-6388, 2017.
24. Fridman WH, Pagès F, Sautès-Fridman C and Galon J: The immune contexture in human tumours: Impact on clinical outcome. Nat Rev Cancer 12: 298-306, 2012.

25. Kanesaka T, Nagahama T, Uedo N, Doyama H, Ueo T, Uchita K, Yoshida N, Takeda Y, Imamura K, Wada K, et al: Clinical predictors of histologic type of gastric cancer. Gastrointest Endosc 87: 1014-1022, 2018.

26. Deng J, Zhang R, Pan Y, Wang B, Wu L, Jiao X, Bao T, Hao X and Liang $\mathrm{H}$ : Comparison of the staging of regional lymph nodes using the sixth and seventh editions of the tumor-node-metastasis (TNM) classification system for the evaluation of overall survival in gastric cancer patients: Findings of a case-control analysis involving a single institution in China. Surgery 156: 64-74, 2014.

27. Feng F, Tian Y, Xu G, Liu Z, Liu S, Zheng G, Guo M, Lian X, Fan D and Zhang H: Diagnostic and prognostic value of CEA, CA19-9, AFP and CA125 for early gastric cancer. BMC Cancer 17: 737, 2017.

28. Mori G, Nakajima T, Asada K, Shimazu T, Yamamichi N, Maekita T, Yokoi C, Fujishiro M, Gotoda T, Ichinose M, et al: Incidence of and risk factors for metachronous gastric cancer after endoscopic resection and successful Helicobacter pylori eradication: Results of a large-scale, multicenter cohort study in Japan. Gastric Cancer 19: 911-918, 2016.

29. Kono Y, Kanzaki H, Tsuzuki T, Takatani M, Nasu J, Kawai D, Takenaka R, Tanaka T, Iwamuro M, Kawano S, et al: A multicenter observational study on the clinicopathological features of gastric cancer in young patients. J Gastroenterol 54: 419-426, 2019.

30. Liu L, Zhang X, Yan B, Gu Q, Zhang X, Jiao J, Sun D, Wang N and Yue X: Elevated plasma D-dimer levels correlate with long term survival of gastric cancer patients. PLoS One 9: e90547, 2014.

31. Kuroda D, Sawayama H, Kurashige J, Iwatsuki M, Eto T, Tokunaga R, Kitano Y, Yamamura K, Ouchi M, Nakamura K, et al: Controlling nutritional status (CONUT) score is a prognostic marker for gastric cancer patients after curative resection. Gastric Cancer 21: 204-212, 2018.

32. Sun KY, Xu JB, Chen SL, Yuan YJ, Wu H, Peng JJ, Chen CQ, Guo P, Hao YT and He YL: Novel immunological and nutritional-based prognostic index for gastric cancer. World $\mathrm{J}$ Gastroenterol 21: 5961-5971, 2015.

33. Liu X, Qiu H, Kong P, Zhou Z and Sun X: Gastric cancer, nutritional status, and outcome. Onco Targets Ther 10: 2107-2114, 2017.

This work is licensed under a Creative Commons Attribution-NonCommercial-NoDerivatives 4.0 International (CC BY-NC-ND 4.0) License. 